\title{
A Multistatic GNSS Synthetic Aperture Radar for Surface Characterization
}

\author{
Tore Lindgren, Student Member, IEEE, and Dennis M. Akos, Senior Member, IEEE
}

\begin{abstract}
Bistatic global navigation satellite system (GNSS) radar has received increased attention in recent years within both the radar and GNSS communities. In this paper, the traditional bistatic GNSS radar and bistatic synthetic aperture radar (SAR) concepts are fused into a more generic multistatic GNSS SAR system for surface characterization. This is done by using the range and Doppler processing techniques on signals transmitted by multiple satellites to determine the angular dependence of the surface reflectivity. The method has also been tested experimentally, and the results are presented.
\end{abstract}

Index Terms-Global positioning system (GPS), radar measurements.

\section{INTRODUCTION}

B ISTATIC and multistatic radars, in which the transmitters and receivers are separated, are useful tools for remote sensing of surfaces. When using global navigation satellite systems (GNSSs) as the source of illumination, there are two main approaches. The first is to make a detailed analysis of the specular reflection. The detailed information about land and sea surfaces in a limited region can then be obtained by analyzing how a surface scatters energy in different directions [1]. The second approach is to use the concept of bistatic synthetic aperture radar (SAR). This is not limited to the specular reflections, making it particularly useful for detecting surface structures and objects [2]. In this paper, these two concepts are fused into a more generic multistatic SAR system for surface characterization, which is also tested experimentally.

Bistatic and multistatic radar systems have certain advantages over a conventional monostatic radar. By making full use of the geometric diversity, detailed information about the properties of a surface can be obtained [1]-[5]. It can also be used as an alternative altimetry system for aircraft and satellites [6]. Because existing signals from other sources, such as television stations and satellites, and monostatic radar transmitters can be utilized, no power needs to be transmitted by such a system, which then can be made smaller and less expensive than a conventional radar. This makes it suitable for applications with a limited power and weight budget [7]. The fact that the radar is completely silent has an obvious advantage from a covert point of view, particularly together with the

Manuscript received May 10, 2007; revised December 10, 2007.

T. Lindgren is with the Department of Computer Science and Electrical Engineering, Luleå University of Technology, 97187 Luleå, Sweden.

D. M. Akos is with the Aerospace Engineering Science Department, University of Colorado at Boulder, Boulder, CO 80309 USA, the Luleå University of Technology, 97187 Luleå, Sweden, and also with Stanford University, Stanford, CA 94305 USA.

Digital Object Identifier 10.1109/TGRS.2008.917268

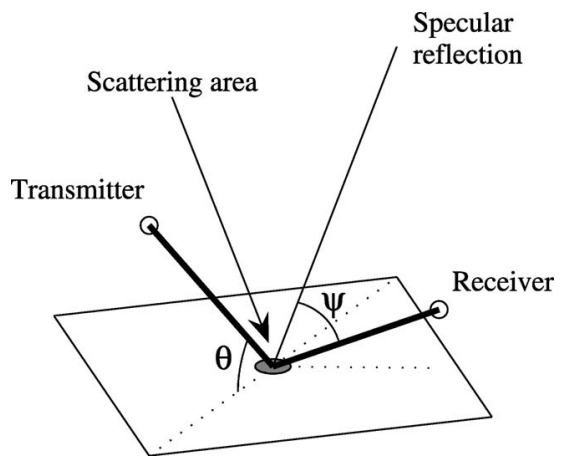

Fig. 1. Bistatic radar geometry. $\theta$ is the elevation angle of the incoming signal, and $\psi$ is the scattering angle, which is defined here as the angle between the direction of the specular reflection and the direction of the observed nonspecular reflection.

geometric-diversity enabling detection of objects designed to make such detection difficult (e.g., stealth aircraft) [8].

The U.S. global position system (GPS) is the only fully operational GNSS and nominally consists of 24 satellites in six orbital planes in a nearly circular (eccentricity of less than 0.02) medium Earth orbit with a semimajor axis of approximately $26560 \mathrm{~km}$ [9]. The major advantages of using GNSS signals for multistatic radar purposes are the global coverage and the time precision nature of the signals. Also, there are usually a large number of satellites visible to the receiver, which increases the geometric diversity of the radar system, making detection of ground features easier. A major challenge is the low power of the signals; the maximum direct-signal level is not expected to exceed $-123 \mathrm{dBm}$ [9], and it will be considerably lower for a reflection. It is, in general, not a problem to detect a specular reflection from the ground or large flat surfaces such as buildings [10]. The main interest of this paper is, however, in the reflectivity of the ground in other directions than specular.

The radar system considered in this paper consists of a set of transmitters in GPS orbit and a receiver located close to the ground. This is shown in Fig. 1. The amount of energy scattered from a surface depends on the scattering direction that is relative to the incident direction, with most energy being scattered in the specular direction. To calculate the energy scattered from a specific area on a surface, both the delay and Doppler properties of the reflection with respect to the direct signal must be analyzed. This is done in Section II. An experiment was carried out at the Boulder Atmospheric Observatory (BAO) outside Boulder, $\mathrm{CO}$, in order to validate the algorithm, with results described in Section III Finally, conclusions are given in Section IV. 


\section{Data Collection And Processing}

The algorithm presented in this paper uses the delay and Doppler properties of the reflected signal to retrieve a maximum amount of information about the observed object or surface. It is largely based on [2] and [11], but it has been adapted to work with longer continuous data sets during which the satellites have time to move considerably. For a general multistatic radar experiment, the following operations are performed.

1) Data collection. Binary GPS data are collected by using a dual-channel software GPS receiver.

2) Low-level processing. Complex correlator waveforms are calculated for the reflected data, and the positions of the receiver and satellites are calculated by using the direct data.

3) Range processing. The correlation waveforms are analyzed with respect to the expected delay of a reflection.

4) Doppler processing. The correlation waveforms are analyzed with respect to the expected Doppler of a reflection that is relative to the direct signal.

\section{A. Data Collection}

In a traditional GPS receiver, all low-level-processing operations (correlation and tracking) are performed in hardware using an application-specified integrated circuit. This solution is very time efficient, and it has been used extensively in bistatic GNSS radar research [12], but it provides limited flexibility. An alternative approach is to use a software GPS receiver where the hardware solution used for the low-level processing is replaced by a programmable processing solution. This way, maximum flexibility is ensured, which is important for multistatic GNSS radar operations where data are recorded on at least two channels in parallel (direct and reflected signals). Particularly useful is the ability to slave the reflected channel to the direct one, obtaining an accurate reference.

The data are recorded by using a dual-channel software GPS receiver. A generic dual-channel software receiver design is shown in Fig. 2. The channel used to record the direct GPS signal uses a right-hand circularly polarized (RHCP) antenna. A left-hand circularly polarized (LHCP) antenna is used for the reflected channel. Both the direct and reflected signals are converted into an intermediate frequency (IF) in separate front ends driven from the same clock. The IF signal is sampled at 16.3676 MHz using 4 bits and stored on a disk for postprocessing. A detailed discussion of the hardware of this receiver can be found in [7].

\section{B. Low-Level Processing}

The signal structure of the GPS system is based on code division multiple access and sent on two different frequencies. For this application, only the civilian GPS signal (coarse/ acquisition or C/A code) in the L1 band (1575.42 MHz) is considered, although there are several more options with existing and future GNSS systems that will have more potential. The C/A code is a 1023-bit (referred to as chips) long pseudorandom-noise (PRN) code that is unique to each satellite, enabling the use of the same frequency for all satellites.

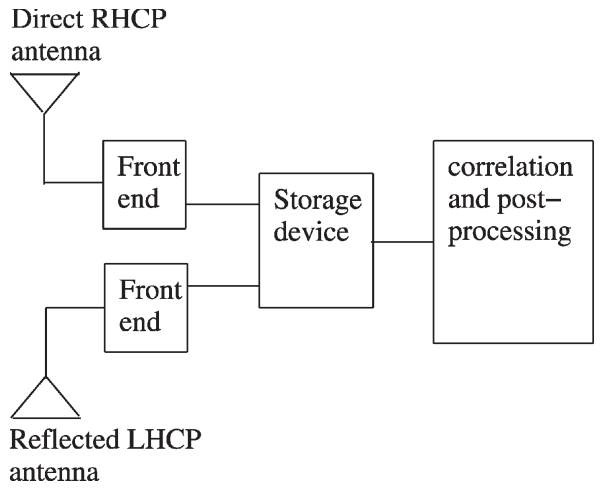

Fig. 2. Data collection setup.

The binary data on the direct channel are processed by using a software receiver. This provides information about the receiver position and time and the satellite position. It also produces a conversion between sample number and time of week. The reflected channel is slaved in time and frequency to the direct channel, i.e., the parameters used when tracking the direct channel are used when calculating the correlator waveform of the reflected channel. In the ideal case, this would have a triangular shape, but due to the limited bandwidth of the hardware used for the data collection (typically $2 \mathrm{MHz}$ for a narrowband L1 front end), it will have a somewhat rounded peak. One correlator waveform is calculated for each millisecond and used in the range and Doppler processing. By slaving the reflected channel to the direct channel, these will automatically be synchronized. Also, large continuous data sets can be processed.

\section{Range Processing}

Due to the low power of the received signal that is expected for nonspecular reflections, a long coherent integration time of the reflected signal is desired in order to reduce the noise. This is typically not a problem because the reflected channel is slaved to the direct. Care must, however, be taken to ensure that the phase of the reflected signal does not change with more than $90^{\circ}$ with respect to the direct. This can be compensated by introducing a phase term $e^{j k \phi}$, where $k$ is the wavenumber and $\phi$ is the phase shift. The coherent sum of $N$ complex valued correlation waveforms can be written as

$$
s(\mathbf{r}, t)=\sum_{\tau=1}^{N} s_{\tau}(\delta, t) e^{j k \phi(\mathbf{r}, \tau)}
$$

where $s_{\tau}(r, t)$ is the correlation waveform at time $\tau$ within epoch $t, \delta$ is the time delay of the reflected signal, and $\mathbf{r}$ is a coordinate in the ground plane. The phase $\phi(\mathbf{r}, \tau)$ also depends on the change in the satellite reflection receiver geometry and not only on the delay of the reflected signal. A different coherent sum should therefore be done for different points on the observed surface. A signal, which is reflected from a surface, that is received by a radar at a height $h$ above the ground and with a delay $\delta$ relative to the direct signal can come from a point $(x, y)$ anywhere on a contour with constant delay (isorange contour). The isorange contour is the intersection of 
the observed surface with the surface of constant delay. The latter is called the isorange ellipsoid and described in [11]. The equation for the isorange contour has been calculated before for a spaceborne receiver with the Earth modeled as an ellipsoidal surface [6]. These calculations are included here for the simplified case when a flat surface can be assumed, which is valid for a receiver at a relatively low height above the ground.

The isorange ellipsoid is given by

$$
\frac{x^{\prime 2}}{b^{2}}+\frac{y^{\prime 2}}{a^{2}}+\frac{z^{\prime 2}}{b^{2}}=1
$$

where

$$
\begin{aligned}
& a=\frac{L+c \delta}{2} \\
& b=\sqrt{a^{2}-\frac{L^{2}}{4}}
\end{aligned}
$$

where $c$ is the speed of light, and $L$ is the distance between the receiver and the transmitter, which are at the foci on the $y$-axis. If the reflection is known to originate from a point that is close to the receiver compared to $L$, which is the case for all nonspaceborne receivers, (2) can be approximated with a paraboloid

$$
\frac{x^{\prime 2}}{2 c \delta}+\frac{z^{\prime 2}}{2 c \delta}=y^{\prime}+c \delta
$$

where the receiver is located at the origin. Rotating this around the $x$-axis with the elevation angle $\theta$ gives

$$
\frac{x^{2}}{2 c \delta}+\frac{(-y \sin \theta+z \cos \theta)^{2}}{2 c \delta}=y \cos \theta+z \sin \theta+c \delta \text {. }
$$

The reflection is assumed to come from a flat surface located at $z=-h$, which gives

$$
\frac{x^{2}}{2 c \delta}+\frac{(-y \sin \theta-h \cos \theta)^{2}}{2 c \delta}=y \cos \theta-h \sin \theta+c \delta .
$$

This can be expanded and simplified to the form

$$
\begin{aligned}
& \frac{x^{2}}{\sin ^{2} 2 \theta}+y^{2}+y A+B=0 \\
& A=\frac{2 h \cos \theta}{\sin \theta}-\frac{2 c \delta \cos \theta}{\sin ^{2} \theta} \\
& B=\frac{h^{2} \cos ^{2} \theta}{\sin ^{2} \theta}+\frac{2 c \delta h}{\sin \theta}-\frac{2 c \delta^{2}}{\sin ^{2} \theta} .
\end{aligned}
$$

Rearranging this into the form $(y-p)^{2}-q$ gives

$$
\begin{aligned}
& \frac{x^{2}}{\alpha^{2}}+\frac{\left(y-y_{c}\right)^{2}}{\alpha^{2} / \sin ^{2} \theta}=1 \\
& y_{c}=(c \delta-h \sin \theta) \frac{\cos \theta}{\sin ^{2} \theta} \\
& \alpha=\frac{\sqrt{c \delta^{2}-2 c \delta h \sin \theta}}{\sin ^{2} \theta} .
\end{aligned}
$$

$h$ is the height above ground, and $\theta$ is the elevation of the satellite transmitting the signal.

\section{Doppler Processing}

The signal $s(\mathbf{r}, t)$ in (1) will be the sum of the signals reflected off the ground along the isorange contour given in (7). The contributions from these reflections will interfere constructively or destructively with the reflection originating from the point $\mathbf{r}$, depending on the relative phase. This mix of signals will produce a Doppler beat frequency which is the difference in Doppler shift between the contributing reflections. The contribution from reflections with different Doppler shifts is found, taking the Fourier transform of the signal in (1)

$$
S(\mathbf{r}, \Delta f)=F\{s(\mathbf{r}, t)\} .
$$

The signal originating from the point $\mathbf{r}$ will have no relative Doppler shift, and it is found by taking the value at $\Delta f=0$. This is equivalent to calculating the average of $s(\mathbf{r}, t)$. The received power $S$ after Doppler processing using $t_{n}$ seconds of data is then

$$
S(\mathbf{r}, 0)=t_{n}^{-1} \int_{0}^{t_{n}} s(\mathbf{r}, t) d t .
$$

The iso-Doppler surface, from which any signal will have the same Doppler shift with respect to the point $\mathbf{r}$, has been calculated in [13] as all points that satisfy

$$
\frac{1}{\lambda}\left[\mathbf{v}_{T} \cdot \mathbf{r}_{T}+\mathbf{v}_{R} \cdot \mathbf{r}_{R}\right]=0
$$

where $\lambda$ is the wavelength of the signal (for the GPS L1 band, it is approximately $19.03 \mathrm{~cm}), \mathbf{v}_{T}$ and $\mathbf{v}_{R}$ are the velocity vectors of the transmitter and the receiver, respectively, and $\mathbf{r}_{T}$ and $\mathbf{r}_{R}$ are the range vectors of the transmitter and the receiver, respectively. Here, a stationary receiver is used, which will eliminate the second term in (10). We then have

$$
\frac{1}{\lambda} \mathbf{v}_{T} \cdot \mathbf{x}=0 .
$$

The isorange and iso-Doppler contours will intersect at the point $\mathbf{r}$ and also at another point unless the iso-Doppler is tangential to the isorange. The power reflected from a small area on the ground can be calculated by using (1) and (8).

\section{BAO TOWER EXPERIMENT}

An experiment was carried out at the National Oceanic and Atmospheric Administration's BAO outside Boulder, CO, on April 4, 2006. The purpose of this experiment is to investigate the potential of using reflected GNSS signals as a multistatic SAR system under more controlled circumstances than what is possible with an airborne experiment. The BAO tower is a 300-m-high tower used for atmospheric and remote sensing research. Data were collected by using a software GPS receiver with a zenith-pointing RHCP antenna and a down-looking fourelement LHCP antenna array pointing at $45^{\circ}$ from nadir. The antenna array provided $15 \mathrm{~dB}$ of gain and also minimized unwanted interference from a direct signal. The IF data (IF frequency $=4.1304 \mathrm{MHz}$ ) were sampled at a frequency of 


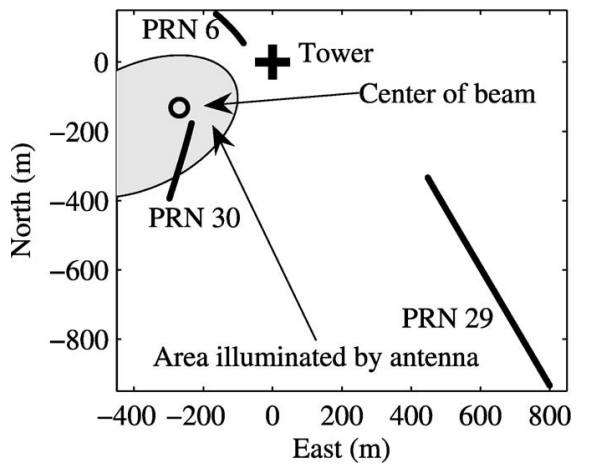

Fig. 3. Specular points of selected satellites in relation to the tower. The coordinate system is a local east-north-up system with the receiver located at the origin.
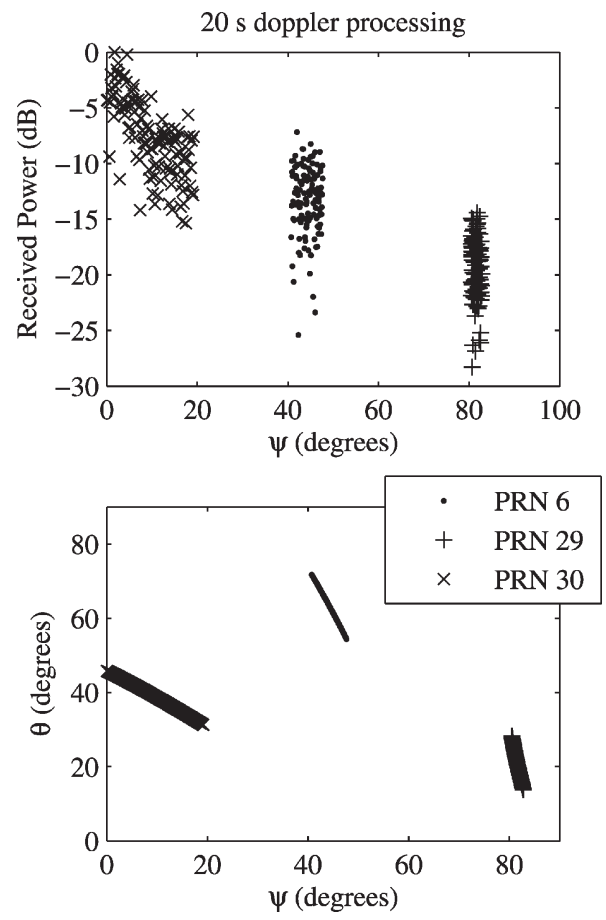

Fig. 4. Measurement results for PRNs 6, 29, and 30 using $20 \mathrm{~s}$ of data for the Doppler processing. The power is normalized to the maximum observed power.

16.3676 MHz using 4 bits/sample. The bandwidth of the receiver is $3 \mathrm{MHz}$.

The data from the direct channel were postprocessed by using a software GPS receiver in order to obtain information about the position of the receiver and the satellites. The data from the reflected channel were processed by using MATLAB to obtain 1-ms correlator dumps. A total of 73 correlators were used between -1.1 and 6.1 chip delay. Three satellites which are chosen for processing with different elevation and bistatic angles were processed (PRNs 6, 29, and 30). The specular points of these satellites, which are relative the tower and the area illuminated by the antenna, are shown in Fig. 3. The range processing was done by using 1 -s coherent integration time.

\section{A. Result}

The results from the measurements are shown in Figs. 4-6 for 20-, 50-, and 100-s Doppler processing time. The received
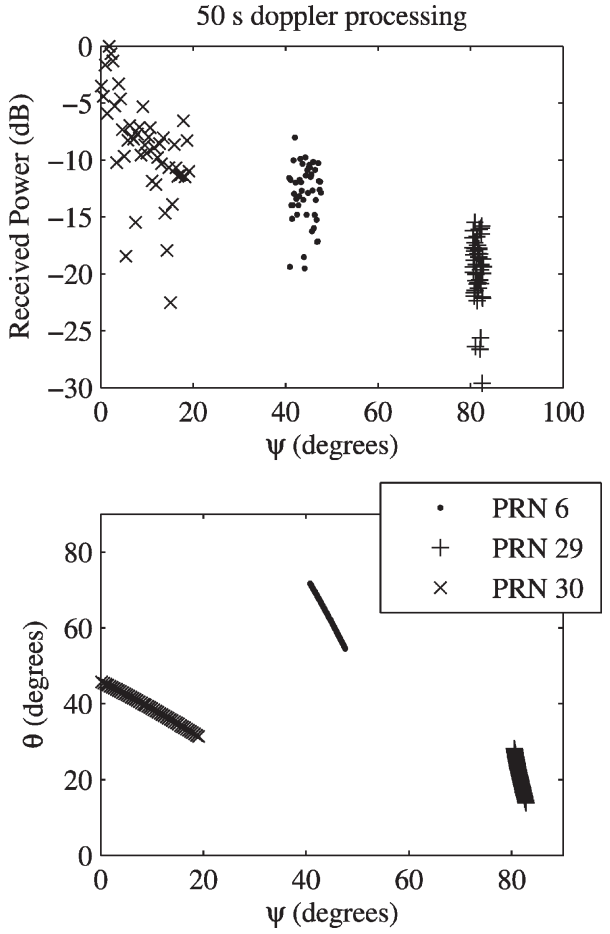

Fig. 5. Measurement results for PRNs 6, 29, and 30 using $50 \mathrm{~s}$ of data for the Doppler processing. The power is normalized to the maximum observed power.
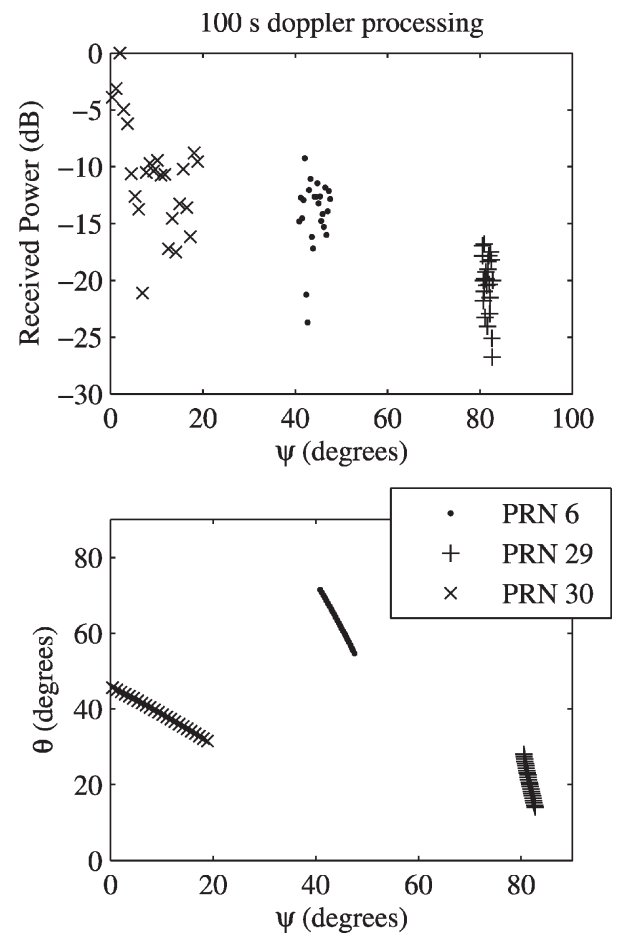

Fig. 6. Measurement results for PRNs 6, 29, and 30 using $100 \mathrm{~s}$ of data for the Doppler processing. The power is normalized to the maximum observed power.

power is shown as a function of the scattering angle $\psi$ from the specular reflection described in Fig. 1. This is a simplified way of presenting the data, because the scatter properties of a surface depend on both the elevation and azimuth of the scatter direction. It is shown here in this way because of the small number of processed satellite signals. 
In all three cases, it can be seen that the received power from PRN 30 is considerably stronger than the power received from both PRNs 6 and 29. This is expected because the reflection from PRN 30 is closer to the specular direction. It can be seen that the reflection from this satellite is significantly stronger and less noisy for the lowest scattering angles. Also, the power received from PRN 6 is somewhat stronger than the one received from PRN 29. This indicates that PRN 6 contains some reflected energy, and it could therefore be used when characterizing the surface. This is uncertain for PRN 29 for which all the seen energy could be due to noise. The results obtained by using longer Doppler processing time (Figs. 5 and 6) are significantly less noisy than the results obtained by using shorter Doppler processing time (Fig. 4). This enables observation of finer structures in the reflected data. Because the resolution of the radar system is increased as the Doppler processing time is increased, a longer processing time also makes it possible to detect smaller spatial variations and irregularities [11]. A long Doppler processing time does, however, require a more accurate knowledge of the receiver position to avoid smearing of the data. This could complicate this type of processing.

\section{CONCLUSION}

The use of GNSS signals as transmitters for a multistatic SAR system has been investigated and demonstrated experimentally. The observed surface can be characterized by studying the power scattered in different directions. By using longer data segments when doing the Doppler processing and calculating the Doppler beat frequency, the resolution of the system can be increased. The results from the BAO tower experiment are encouraging because they demonstrate that it is possible to use the multistatic SAR concept to the determined properties of a surface. This enables the use of reflected GNSS signals for remote sensing in environments where a nonuniform surface otherwise limits its use.

Further work includes the use of more satellites and different signals and frequencies. Of particular interest is the upcoming launch of the European GALILEO system and the new L2c signal on the modernized GPS satellites. Also, multistatic GNSS SAR should be studied by using dynamic platforms such as airplanes or high-altitude balloons.

\section{ACKNOWLEDGMENT}

The authors would like to thank the National Oceanic and Atmospheric Administration for making the experiment at the BAO tower possible.

\section{REFERENCES}

[1] D. Masters, "Surface remote sensing applications of GNSS bistatic radar: Soil moisture and aircraft altimetry," Ph.D. dissertation, Dept. Aerosp. Eng. Sci., Boulder, CO, 2004.

[2] M. Cherniakov, R. Saini, R. Zou, and M. Antoniou, "Space surface bistatic SAR with space-borne non-cooperative transmitters," in Proc. IEEE EURAD, Paris, France, 2005, pp. 9-12.

[3] M. Armatys, D. Masters, A. Komjathy, P. Axelrad, and J. L. Garrison, "Exploiting GPS as a new oceanographic remote sensing tool," in Proc. ION NTM, Long Beach, CA, 2001, pp. 339-347.
[4] R. Sabia, M. Caparrini, A. Camps, and G. Ruffini, "Potential synergetic use of GNSS-R signals to improve the sea-state correction in the sea surface salinity estimation: Application to the SMOS mission," IEEE Trans. Geosci. Remote Sens., vol. 45, no. 7, pp. 2088-2097, Jul. 2007.

[5] E. Cardellach, G. Ruffini, D. Pino, A. Rius, A. Komjathy, and J. Garrison, "Mediterranean balloon experiment: Ocean wind speed sensing from the stratosphere, using GPS reflections," Remote Sens. Environ., vol. 88, no. 3, pp. 351-362, Dec. 2003.

[6] M. Martin-Neira, "A passive reflectometry and interferometry system (PARIS)-Application to ocean altimetry," ESA J., vol. 17, no. 4, pp. 331-355, 1993.

[7] S. Eterhuizen and D. M. Akos, "The design, construction, and testing of a modular GPS bistatic radar software receiver for small platforms," in Proc. ESA/ESTEC GNSSR: Workshop GNSS Reflections, Noordwijk, The Netherlands, Jun. 2006.

[8] Ø. Overrein, K. E. Olssen, S. Johnsrud, P. K. Sørnes, T. Johnsen, J. Navarro, V. Sahajpal, and R. O. Stemland, "Geometrical and signal processing aspects using a bistatic hitchhiking radar system," in Proc. IEEE Radar Conf., Arlington, VA, May 2005, pp. 332-336.

[9] E. Kaplan and C. Hegarty, Understanding GPS: Principles and Applications, 2nd ed. Norwood, MA: Artech House, 2006.

[10] T. Lindgren, E. Vinande, D. Akos, D. Masters, and P. Axelrad, "Measurement of backscattered GPS signals," in Proc. IEEE/ION PLANS Conf., San Diego, CA, Apr. 2006, pp. 664-669.

[11] N. J. Willis, Bistatic Radar. Norwood, MA: Artech House, 1991.

[12] O. Nogués-Correig, E. Cardellach Galí, J. Sanz Campderrós, and A. Rius, "A GPS-reflections receiver that computes Doppler/delay maps in real time," IEEE Trans. Geosci. Remote Sens., vol. 45, no. 1, pp. 156-172, Jan. 2007.

[13] G. P. Cardillo, "On the use of the gradient to determine bistatic SAR resolution," in Proc. IEEE AP-S Int. Symp., Dallas, TX, May 1990, pp. 1032-1035.

[14] D. Masters, P. Axelrad, V. Zavorotny, S. J. Katzberg, and F. Lalezari, "A passive GPS bistatic radar altimeter for aircraft navigation," in Proc. ION GPS, Salt Lake City, UT, 2001.

[15] T. Zeng, M. Cherniakov, and T. Long, "Generalized approach to resolution analysis in BSAR," IEEE Trans. Aerosp. Electron. Syst., vol. 41, no. 2, pp. 461-473, Apr. 2005.

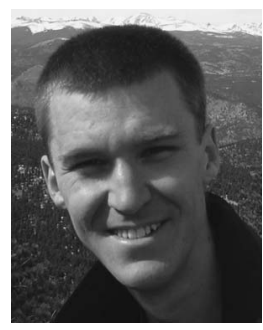

Tore Lindgren (S'07) received the M.Sc. degree in space engineering and the Licentiate degree in electrical engineering from the Luleå University of Technology, Luleå, Sweden, in 2004 and 2007, respectively, where he is currently working toward the Ph.D. degree in electrical engineering in the Department of Computer Science and Electrical Engineering.

His research interests include multistatic global navigation satellite system radar and antenna technology for radar and radio-frequency identification

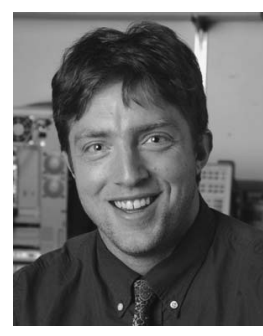

Dennis M. Akos (S'90-M'97-SM'05) received the Ph.D. degree in electrical engineering from Ohio University, Athens.

$\mathrm{He}$ is currently an Assistant Professor with the Aerospace Engineering Science Department, University of Colorado, Boulder, and holds a Visiting Professor Appointment with Luleå University of Technology, Luleå, Sweden, and a Consulting Professor Appointment with Stanford University, Stanford, CA. His research interests include global navigation satellite systems, software defined radio, applied/digital signal processing, and radio-frequency design. 